\title{
Mobile Cloud Service Selection using Back Propagation Neural Network
}

\author{
Arif Ahmed \\ National Institute of Technology, Silchar \\ Silchar, India
}

\author{
Abadhan S Sabyasachi \\ Hongkong University of Science \& Technology, Hongkong \\ Clear Water Bay, Hong Kong
}

Ananya Choudhury
Sardar Vallabhbhai National Institute of Technology, Surat

Surat, India

\begin{abstract}
Cloud computing is a paradigm in high performance computing, focuses on provisioning ubiquitous computing with the help of Software and/or Hardware Virtualization. In Mobile Cloud Computing (MCC), mobile/portable devices access cloud resources through wireless communication(GPRS/3G/WiFi etc). MCC enhances the mobility of the cloud user which solves cloud computing issues such as Unreliability, Quality-of-Service (QoS), etc. Recently QoS has emerged as a one of the challenging issue in MCC which impact to the large number of mobile users and businesses. The QoS in MCC degrades mainly due to its limited bandwidth, network congestion, user mobility, etc. In this paper, we have proposed a mobile cloud computing framework that facilitates the mobile client to access cloud services with a high degree of QoS based on the network condition of the connection. We proposed a new QoS based mobile cloud computing framework . Back Propagation Neural Network (BPNN) is being used for predicting and selecting appropriate cloud service for the mobile client. We have implemented the proposed framework taking QoS parameters: Packet Delivery Ratio (PDR), Transmission Rate, and Delay in a mobile cloud computing environment. At the end, we have compared our model with the random selection approach and it shows that the proposed model gives better performance.
\end{abstract}

\section{General Terms}

Mobile Cloud Computing, QoS

\section{Keywords}

Cloud Computing, Mobile Cloud Computing, Back Propagation Neural Network, QoS, Cloud Service

\section{INTRODUCTION}

Cloud computing, a model of utility computing, enhances end user availability of different computing resources, operating platform/or application software of the cloud service on pay-as-you-go model[1]. The key idea of cloud computing differs from the grid computing is the unique service level of abstraction and virtualiza- tion[16]. Cloud computing is becoming more popular for the multiple commercial significant e.g. less initial investment, fast start-up to the new services, low maintenance and security overhead, speedy recovery etc. In general, services in cloud computing can be divided into Infrastructure as a Service(IaaS), Platform as a Service(PaaS), Software as a services(SaaS).

The end-user can access cloud services using Desktop and other portable devices (such as Mobile phone, Tablet etc.). The extension of cloud computing to the mobile computing with wireless interface is referred as Mobile Cloud Computing [2]. Mobile Cloud users utilizes the resources of both cloud computing and mobile computing. The term mobile cloud computing can be defined as "A service that allows resource constrained mobile users to adaptively adjust processing and storage capabilities by transparently partitioning and offloading the computationally intensive and storage demanding jobs on traditional cloud resources by providing ubiquitous wireless access"[3]. According a survey conducted in US, the number of internet users from mobile devices will be out number from the desktop computer [5]. In mobile cloud computing, cloud computing services are available into mobile or portable devices through wireless interface(e.g. GPRS/3G/WiFi). In the above scenario, user utilizes both mobility and cloud resources, despite lots of problem arose such as 1 . Scarcity of resource, 2 . Weak battery strength, 3 . User mobility 4. Low network coverage, 5. QoS Management[9]. Quality of Service(QoS) referred as requirement of non-functional attributes that affect the performance in a service most specifically network research community[6]. Likewise in many applications of mobile cloud computing, Quality-of-service remains one of the vital challenging from the above paragraph. According to International ISO 840 , Quality is a totality of features and characteristics of a merchandise or services that bear the ability to satisfy the requirement stated or implied needs[7]. In other words, QoS is a set of non-functional values that influence Quality of Service of a service offered by an application. QoS in Mobile Cloud Computing can be illustrated as how a cloud provider ensures its QoS to its customer. QoS in MCC can be achieved in various ways: QoS in run time, Security related QoS, price-QoS Trade-off etc[8].

Here, in throughout the paper, QoS is referring to dynamic QoS properties such as packet loss ratio, end-to-end throughput, delay etc. in the wireless network environment of mobile network. In the recent years, some existing work have been done on above issue 


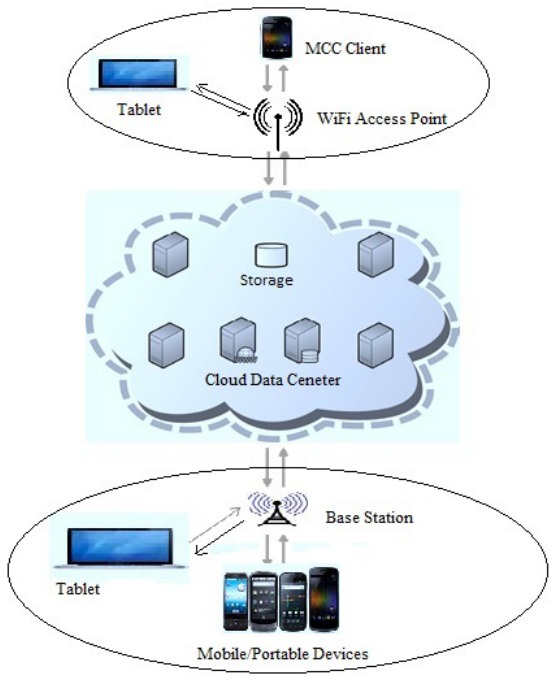

Fig. 1: Mobile Cloud Computing Architecture[8]

in MCC. But, there is a lack of incomplete remains in related with inaccessibility, service discovery, resource constrains and user mobility. ${ }_{i} b_{i}$

In this paper, we present a mobile cloud model to provide cloud services at the end-user with maximum QoS according to the dynamic network environment. This model has a mobile terminal monitor that tracks the network condition according to which cloud will provide the appropriate service. This paper is structured as follows : Literature survey of the work is described in section 2 . The proposed model/framework and algorithms are described in Section 3. Section 4 illustrate how the experimentation of the proposed model is carried. The result and analysis of the experimentation is done in section 5. The conclusion and future direction of this paper is made in the last section.

\section{LITERATURE SURVEY}

Research in MCC is mainly focused on operational level, service level, end-user level, context aware management, privacy and security[4]. Performance issue for a cloud service is determined by the availability of the service and QoS of the system. According to recent survey on QoS issue in Mobile Cloud Computing(MCC), There are few research works have been carried out on this issues but most of the problem are still open and QoS can be dealt with in three ways: Runtime related QoS, Security and security[8]. In[10] , a QoS based mobile cloud computing framework is proposed,and Fuzzy Cognitive Map is used to map appropriate cloud service at the end-user. Wang et al. proposed a QoS based architecture for cloud to provide video on demand services[11]. In Ye et al. a new model is proposed for QoS and power management(QPM) during service selection in mobile cloud computing environment[12]. The main goal of QPM framework is to reduce the power consumption in mobile terminal with high QoS. But we have seen most of the above works has not considered important network based QoS parameters for the service selection process and use of neural network.

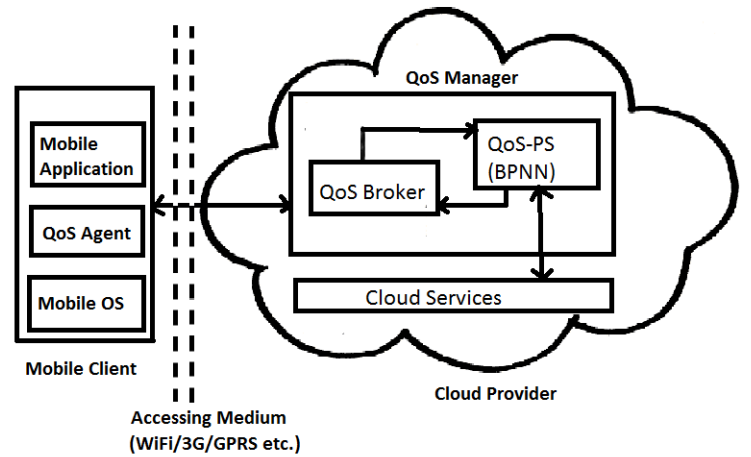

Fig. 2: Proposed Mobile Cloud Architecture for Service Selection

\section{PROPOSED MODEL}

Our goal is to facilitate cloud service at the mobile environment for each request after checking communication status of the network. The network attributes are dynamically capture at the mobile host and send back to the cloud service along with the request. We define each entity involved in the process as follows:

1.Cloud services(S): We have defined the cloud service/application with a set of service from low to high quality of services. Let $\mathrm{S}=\left\{s_{1}, s_{2}, s_{3}, . s_{n},\right\}$ is a set of service set where $\mathrm{n}$ is the number of service provided by the service provider.

2. QoS Attributes(A): These are the attributes of network status i.e. QoS parameter involves in determining the service to be provide from the pool of service. Assuming $\mathrm{A}=\left\{a_{1}, a_{2}, a_{3}, . . a_{r}\right\}$ is the set having attributes the collect by the network host.

3. Service Criteria(C): For each service, there are certain set of rules agreed between service provider and end-user about the services. Let, $\mathrm{C}=\left\{c_{1}, c_{2}, c_{3}, \ldots, c_{i}\right\}$ is a set of criteria where $i \geq n$.

4. Input parameter(I): when a mobile host sends a request for service, it attached QoS parameters(A) along with the request. The cloud end decode these values and provide the appropriate service from based on the result of QoS measurement function.

\subsection{Proposed Model and Components}

The model Mobile Cloud Service Selection framework is shown in the figure 2. The model utilizes back propagation neural network in choosing the best service from the poll of service. It framework has four components:

1. QoS Agent: It has the responsibility to send QoS parameters from the mobile to cloud when the request is made. QoS agent dynamically captures parameters and attached to the request.

2. QoS Broker: QoS Broker receives the request at the cloud end, de-code the request and extract QoS parameters. After that, It searches the requested service and if available, QoS parameters are used for right prediction QoS prediction Service(QoS PS).

3. QoS Prediction Service(QoS PS): This module takes QoS parameters as input and reply with the appropriate service. The service is then send to the Mobile clinet. QoS PS is implemented with Back Propagation Neural Network(BPNN) that used for prediction from the trained data.

4. Cloud Service: Cloud Services are the set of services for each application. For each service, a training set is developed to train the pattern in BPNN. 


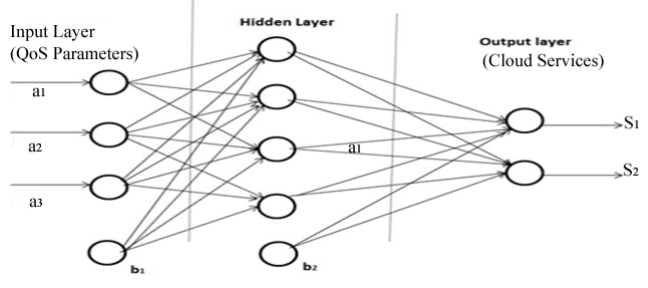

Fig. 3: A 4-5-2 Back-Propagation Neural Network

\subsection{Back Propagation Neural Network}

Artificial Neural Network is biologically inspired computational model. . Back propagation neural network is one of the biological inspired neural networks used for service prediction, classification in various field $[7,13]$. Back propagation neural network is a popular and widely used artificial neural network. It is multi-layer feed forward network, at first trained according to trained data. BPNN is used in machine learning and deals with the mapping of large number of input-output models. It uses the method of mean square error (mse) and gradient descent to update the connection weight of the network at the end-of a training. The weight is updated to minimize the error sum of square.

\subsection{Algorithm}

There are two algorithms involved in the model that are related with the back propagation neural network. First, the training algorithm which carried out at the initiation of the working mode. After getting the proper input(combination of QoS parameters and service) and each input set is trained to the BPNN using this algorithm. Second, once training of all the training data set are performed, the testing algorithm is used to select the best service according to the QoS value from the QoS input.

In our case, if we represent train data in a matrix form then it can be represented in following way

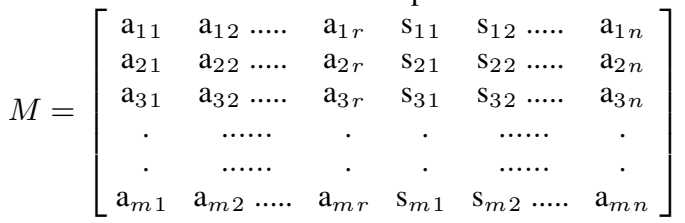

3.3.1 Algorithm to train the BPNN. Input: $\mathrm{C}$, a data set consisting of the training tuples and their associated target values; 1 , the learning rate; network, a multilayer feed-forward network.

Output: A trained neural network.

(1) Initialize all the weights and biases in network;

(2) while the terminating condition is not satisfied \{

(3) for each training tuple "c" in M \{

(4) // Propagate the i/p forward:

(5) for each input layer unit $\mathrm{j}\{$

(6) $\quad O_{j}=a_{j} ; / /$ output of an i/p unit is its actual $\mathrm{i} / \mathrm{p}$ value

(7) for each hidden oro/p layer unit $\mathrm{j}\{$

$$
I_{j}=\sum_{i} W_{i j} O_{i}+\theta_{j}
$$

\} //compute the net $\mathrm{i} / \mathrm{p}$ of unit $\mathrm{j}$ with respect to the previous layer, $\mathrm{i}$
(9)

// to the first hidden layer

(14)

layer, $\mathrm{k}$

(15) for each weight $w_{i j}$ in network \{

$$
\Delta W_{i} j=(l) E r r_{j} o_{j}
$$

//weight increment

$$
W_{i j}=W_{i} j+\Delta W_{i} j
$$

//weight update

for each bias $\Theta_{j}$ in network \{

$$
\Delta \theta_{j}=(l) E r r_{j}
$$

//bias increment

$$
\theta_{j}=\theta_{j}+\Delta \theta_{j}
$$

//bias update

\} \}

3.3.2 Algorithm to find Appropriate Service. Input: QoS Attribute set $\mathrm{A}=\left\{a_{1}, a_{2}, a_{3},-----, a_{r}\right\}$ for a particular received from the QoS Agent.

Neural Network: network

Weights and biases values in the network

Output: $S_{j}$ service from the set of services.

(1) Check whether service is available if no, no service, otherwise goto 2

(2) for each input layer unit $\mathrm{j}\{$

(3) $o_{j}=a_{j}$;//output of an input unit is its actual input value

(4) for each hidden or output layer unit $\mathrm{j}\{$

$$
I_{j}=\sum_{i} W_{i j} O_{i}+\theta_{j}
$$

compute the net input of unit $\mathrm{j}$ with respect to the previous layer,

(6)

$$
\left.o_{j}=1 /\left(1+e^{(}-I_{j}\right)\right)
$$

(7) compute the output of each unit $j$

(8) Choose the greatest value $o_{j}$ from the all the unit and jth is the output service. 
Table 1. : Data Set collected for training

\begin{tabular}{|l|l|l|}
\hline PDR & $\begin{array}{l}\text { Transmission } \\
\text { Rate(MB/S) }\end{array}$ & Delay(Sec.) \\
\hline 0.996 & 5.216 & 0.003015461 \\
\hline 0.996 & 5.226 & 0.003015461 \\
\hline 0.896 & 4.220 & 0.009015461 \\
\hline 0.880 & 4.000 & 0.01015461 \\
\hline 0.800 & 3.216 & 0.103015461 \\
\hline
\end{tabular}

\section{EXPERIMENTATION}

In [17], a survey on cloud computing simulators is carried out, and there is no suitable simulator available to simulate above proposed model. The model is implemented in a mobile cloud environment and experimentation is done in varying network bandwidth. A separate application is developed in android to calculate profiler i.e. runtime QoS parameters such as network bandwidth, Packet Lost and delay. The back propagation neural network is implemented in python, and data set are stored in .csv fil. The experimentation is carried out in different network edge namely gprs, 3G and Wifi environment.

There are three QoS attributes used in the experimentation model and they are:

a) Packet Delivery Ratio (PDR)(p): It gives the information about the packet loss during the communication. In general, Packet delivery ratio (PDR) can be defined as the ratio of the number of delivered data packet to the destination.

b) Transmission Rate(t): Transmission rate provides the amount of data transfer during the communication in 1 second.

c) Delay(d): The average time taken by a data packet to arrive in the destination. It also includes the delay caused by route discovery process and the queue in data packet transmission. Only the data packets that successfully delivered to destinations that counted.

We have considered are three services on the model: S1, S2 and S3. where

a) $\mathrm{S} 1=$ High service configuration with high cost

b) $\mathrm{S} 2=$ Medium service configuration with medium cost

c) $\mathrm{S} 3=$ Low service configuration with low cost

During the experimentation, packets(each size 256kb) are sent from the mobile terminal(QoS Agent)to the cloud. For each request, at the cloud service provider, QoS broker accept the request and response it immediately. Mobile terminal can calculate all the QoS parameters from the request. We have used Android API to develop QoS Agent. In this setup, QoS agent preform the request after 5 second periodically. The experimentation is carried in both $\mathrm{WiFi}$ and 3G. Cloud server is created using VirtualBox 4.0.32[14, 15], which used type- 2 hypervisor. After carrying out the experimentation, we have defined QoS range for the services and defined the training table(SLA). We have collected 2000 number of data set to be used in our framework for service selection. Now, the QoS profile is dived into three services based on the packet delivery ratio. We have shown 5 dataset in table. Table 1 shows a sample of training data.

After collecting the data set, the next step is to build a back propagation neural network according to the configuration requirement of our framework. As we have consider 3 QoS attributes and 3 services, we need to create a back propagation neural network with
Table 2. : Back Propagation Neural Network Configuration.

\begin{tabular}{|l|l|}
\hline Property & Values \\
\hline No. of input & 3 \\
\hline No. of output & 3 \\
\hline No. of hidden nodes & 7 \\
\hline Learning Rate & 0.05 \\
\hline Maximum Iteration & 2000 \\
\hline $\begin{array}{l}\text { Mean Square Error stopping condi- } \\
\text { tion }\end{array}$ & 0.0001 \\
\hline
\end{tabular}

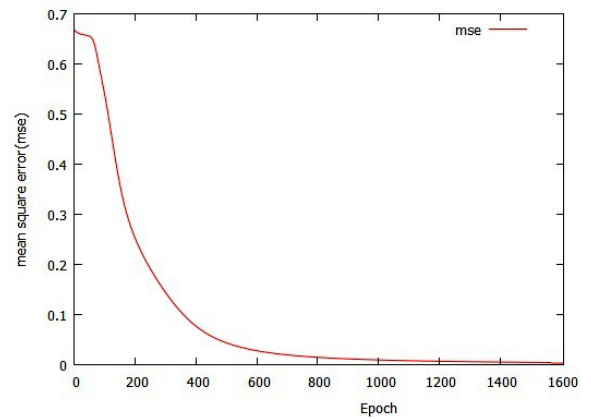

Fig. 4: NEURAL NETWORK TRAINING ERROR

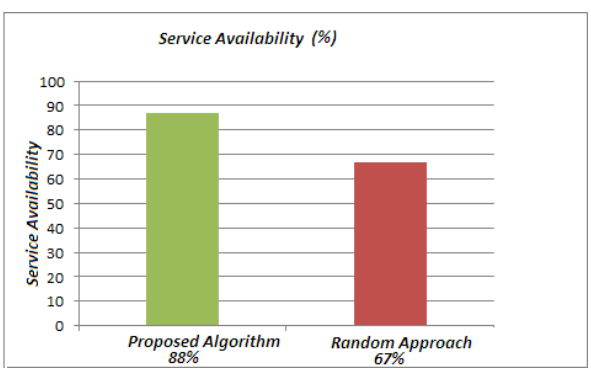

Fig. 5: Performance Comparision with random approach

3 inputs and 3 output. Configuration for Back Propagation Neural Network Configuration in the setup is shown in table 2 .

\section{RESULT \& ANALYSIS}

The overall performance of the proposed framework can be evaluated by in two ways: how accurately service is predicting by the back propagation neural network and in the second case, the time complexity of the back propagation neural network. We can check the number of mean square error value with the number of iteration, so that we can stop the iteration when the mean square error value is at constant. If it take more iteration then the computation takes to trained the collected data is high. We can also compare the performance of our algorithm with other service mode i.e. randomly approach where service selection is done randomly. Random approach takes QoS parameters and randomly choose a particular service and send it to the mobile client. To check the performance, we are evaluating number of available services for a time being for both cases and compare the availability.

The availability is calculated in percentage (Service Available = (Total no. of Service Response/Total no. of Service Requested) * 
$100 \%)$. We found that the service availability in our proposed algorithm is $88 \%$ and with that of random approach it is $67 \%$. As we can see that, availability of services is more than the random approach. From here we can conclude that the proposed technique give better performance from the random model.

\section{CONCLUSION}

Mobile Cloud Computing is one of the important topics in cloud computing due to most of cloud user as accessed from the mobile devices. In general, mobile devices access cloud services via wireless communication medium (such as GPRS, 3G/Wi-Fi etc.). Mobile cloud computing brings a lot advantages to the both mobile terminal and business entrepreneur through virtualization and different level of service abstraction. Although, challenges in mobile cloud computing are also not limited. Mobile Cloud Computing inherits the challenges of mobile terminal and that are the in cloud component and barriers in between them(i.e. communication medium). Quality-of-service is also one of the many challenging aspects of mobile cloud computing and high QoS of a particular service can be achieved in different way as its depend on the various component. We have proposed a new QoS based service selection model with the use of back propagation neural network. The proposed model is implemented real mobile cloud computing environment. The proposed model can be extended by taking other parameters such as availability, reliability, cost etc.

\section{REFERENCES}

[1] P. Mell and T. Grance : , The NIST Definition of Cloud Computing, National Institute of Standards and Technology, vol.53, no.6, p.50, 2009. [Online]. Available: http://csrc.nist.gov/groups/SNS/cloudcomputing/ clouddef-v15.doc..

[2] Dinh, H. T., Lee, C., Niyato, D., \& Wang, P. A survey of mobile cloud computing: architecture, applications, and approaches, Wireless communications and mobile computing, 13(18), 1587-1611, 2013.

[3] Khan, A. N., Kiah, M. M., Khan, S. U., \& Madani, S. A., Towards secure mobile cloud computing: A survey, Future Generation Computer Systems, 29(5), 1278-1299, (2013)..

[4] Fernando, N., Loke, S. W., \& Rahayu, W., Mobile cloud computing: A survey, Future Generation Computer Systems, 29(1), 84-106, (2013).

[5] http://www.pewinternet.org/2015/04/01/us-smartphone-usein-2015/.

[6] Ran, S. , A model for web services discovery with QoS , ACM Sigecom exchanges, 4(1), 1-10, (2003).

[7] Boritz, J. E., \& Kennedy, D. B. Effectiveness of neural network types for prediction of business failure. , Expert Systems with Applications, 9(4), 503-512, (1995).

[8] Ahmed A., Sabyasachi A. S. \& Barlaskar E., A survey on the QoS issue of Mobile Cloud Computing and Future Direction, 8th International Conference on Communication Network, pp. 248255(2014).

[9] Sanaei, Z., Abolfazli, S., Gani, A., \& Buyya, R., Heterogeneity in mobile cloud computing: taxonomy and open challenges, Communications Surveys \& Tutorials, 16(1), 369-392, (2014).

[10] Zhang, P., \& Yan, Z. A QoS-aware system for mobile cloud computing, IEEE International Conference on Cloud Computing and Intelligence Systems (CCIS), 2011 (pp. 518-522), (2011, September).
[11] Wang, X., Du, Z., Liu, X., Xie, H., \& Jia, X. An adaptive QoS management framework for VoD cloud service centers, IEEE International Conference In Computer Application and System Modeling (ICCASM), 2010 (Vol. 1, pp. V1-527), 2010, (October).

[12] Ye, Y., Jain, N., Xia, L., Joshi, S., Yen, I., Bastani, F.,\& Bowler, M. K. , A framework for QoS and power management in a service cloud environment with mobile devices, In IEEE Fifth IEEE International Symposium on Service Oriented System Engineering (SOSE), (pp. 236-243), 2010 (June).

[13] Tam, K. Y., \& Kiang, M. Y., Managerial applications of neural networks: the case of bank failure prediction, Management science, 38(7), 926-947, (1992).

[14] VirtualBox: http://www.virtualbox.org/

[15] sberg, M., Forsberg, N., Nolte, T., \& Kato, S. ,Towards realtime scheduling of virtual machines without kernel modifications, In 2011 IEEE 16th Conference on Emerging Technologies \& Factory Automation (ETFA), (pp. 1-4) (2011, September).

[16] Foster, I., Zhao, Y., Raicu, I., \& Lu, S., Cloud computing and grid computing 360-degree compared In Ieee Grid Computing Environments Workshop, 2008 (pp. 1-10). (2008, November).

[17] Ahmed, A., \& Sabyasachi, A. S., Cloud computing simulators: A detailed survey and future direction, In 2014 IEEE International Advance Computing Conference (IACC), (pp. 866872). (2014, February) 